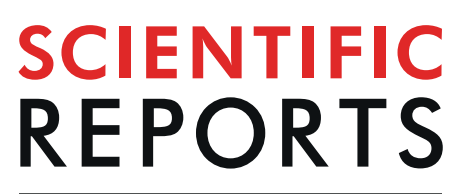

natureresearch

\title{
OPEN Electroosmotic flow driven microfluidic device for bacteria isolation using magnetic microbeads
}

Received: 5 December 2018

Accepted: 31 July 2019

Published online: 02 October 2019

\begin{abstract}
Samuel Miller ${ }^{1}$, Alison A. Weiss $\mathbb{B}^{2}$, William R. Heineman ${ }^{3}$ \& Rupak K. Banerjee ${ }^{4}$
The presence of bacterial pathogens in water can lead to severe complications such as infection and food poisoning. This research proposes a point-of-care electroosmotic flow driven microfluidic device for rapid isolation and detection of $E$. coli in buffered solution (phosphate buffered saline solution). Fluorescent $E$. coli bound to magnetic microbeads were driven through the microfluidic device using both constant forward flow and periodic flow switching at concentrations ranging from $2 \times 10^{5}$ to $4 \times 10^{7}$ bacteria $/ \mathrm{mL}$. A calibration curve of fluorescent intensity as a function of bacteria concentration was created using both constant and switching flow, showing an increase in captured fluorescent pixel count as concentration increases. In addition, the use of the flow switching resulted in a significant increase in the capture efficiency of $E$. coli, with capture efficiencies up to $83 \% \pm 8 \%$ as compared to the constant flow capture efficiencies (up to $39 \% \pm 11 \%$ ), with a sample size of $3 \mu \mathrm{L}$. These results demonstrate the improved performance associated with the use of the electroosmotic flow switching system in a point-of-care bacterial detection assay.
\end{abstract}

The development of micro total analysis systems ( $\mu$ TAS), often referred to as lab-on-chip devices, have ever evolving technological requirements to overcome the difficulties inherent in performing rapid biological assays in a small portable device ${ }^{1}$. The $\mu$ TAS devices are designed to utilize micro- to nano-liter samples while still providing reliable and swift analysis for point-of-care diagnostics. The difficulty of using these devices in a biological assay stems from the need to downscale laboratory process, such as biomolecular targeting, spectroscopy, and image analysis, that requires large equipment and long wait times, into a portable and quick-response system ${ }^{2}$. Typical bacterial toxicity levels range from $10^{6}$ to $10^{8}$ bacteria $/ \mathrm{mL}$, with some particularly infectious strains reporting infectious doses of less than 1000 bacteria/ $\mathrm{mL}^{3}$.

Magnetophoretic based immunoassay is a popular separation technique used in $\mu$ TAS devices. This technique utilizes magnetic microbeads (mMBs) coated with binding ligands that will bind to specific biomolecules so that the mMB-complex can be isolated in the system ${ }^{4-7}$. This separation is accomplished by applying a magnetic field to the system to isolate, or capture, the mMBs and, therefore, the biomolecules that are bound to them. A high capture efficiency, or the ratio between captured beads and the total beads in the system, is essential to the minimization of error in a microfluidic device. The use of $\mathrm{mMBs}$ is ideal for this application due to the ease at which they are isolated in the system using the external magnetic field, their high surface area-to-volume ratio that allows for availability of ample binding sits, and their ability to be adapted to target a multitude of different biomolecules $^{8-10}$. These factors make magnetophoretic separation using mMBs a promising choice for use in a $\mu \mathrm{TAS}$ device that is both reliable and versatile in its application.

\footnotetext{
${ }^{1}$ Department of Mechanical and Materials Engineering, University of Cincinnati, 598 Rhodes Hall, University of Cincinnati, Cincinnati, OH, 45221, USA. '2Department of Molecular Genetics, Biochemistry \& Microbiology, University of Cincinnati, 2254 Medical Sciences Building, 231 Albert Sabin Way, Cincinnati, OH, 45267, USA. ${ }^{3}$ Department of Chemistry, University of Cincinnati, 120 Crosley Tower, PO Box 210172, Cincinnati, OH, 45221, USA. ${ }^{2}$ Department of Mechanical and Materials Engineering, University of Cincinnati, 593 Rhodes Hall, ML 0072, University of Cincinnati, Cincinnati, $\mathrm{OH}, 45221$, USA. Correspondence and requests for materials should be addressed to R.K.B. (email: rupak. banerjee@uc.edu)
} 
Literature review and background. A limited number of studies have been done examining the use of magnetophoretic separation of mMBs. Thompson et al. (2010) studied how the incorporation of microwells affect capture of mMBs in a microfluidic channel, reporting a low capture efficiency of around $13 \%{ }^{11}$. A study by Di Carlo et al. (2007) aims to use inertial focusing in the microchannel in an attempt to improve capture efficiency ${ }^{12}$. This process uses a series of s-shaped bends in a microchannel that organize the mMBs into a single flow line to make them easier to isolate at the outlet. Li et al. (2013) further evaluated how adjusting streamlines in a channel by adding obstacles in the flow path affect capture of particles ${ }^{13}$. Additional changes in channel geometry were part of a numerical study that aimed at filtering based on particle size by Zhang et al. $(2014)^{14}$. A numerical study by $\mathrm{Wu}$ et al. (2011) attempted to predict how capture efficiency changes between a straight channel, L-shaped channel, and T-shaped channel ${ }^{15}$. A study by Beyor et al. (2008) used special pumps to create a pulsatile flow in the channel ${ }^{6}$. Using these pumps, the fluid was driven back and forth through the channel, showing improved capture efficiency up to $70 \%$ due to the multiple passes.

In addition to analysis of channel geometry and flow, how changes in magnetic field in the channel affects mMB capture has also been studied. Munir et al. (2009) performed a numerical study analyzing how the variation of magnet position and number of magnets around a circular chamber in a microchannel affect the capture efficiency of $\mathrm{mMB}^{16}$. A study by Hoshino et al. (2011) explored alternating magnetic polarities along a channel in an attempt to separate mMBs and cancer cells in blood samples ${ }^{17}$. Ramadan et al. (2009) examined whether the use of electromagnets allows for better control and isolation of $\mathrm{mMBs}^{18}$. However, this study created the microchannel using a silicon wafer instead of transparent glass slide while using a high-pressure syringe pump to drive the flow.

The previous work on $\mathrm{mMB}$ capture in microfluidic channels has been performed on a few different channel and magnet configurations. However, most of the studies were numerical in nature while the majority of the experimental studies utilize pressure driven flow instead of electroosmotic flow (EOF) to drive the fluid through the channel. The use of pumps on such smaller scales is expensive and complicated. In addition, the extremely high pressure necessary to pump the fluid through the small microchannels often causes leaking at the junction between the channel and the pump as well as seepage at the bonds between the polymer and glass slide.

The isolation of pathogens in samples using various methods has also been analyzed. Kwon et al. (2008) used streptavidin coated mMBs tagged to fluorescent antibodies to detect toxins in a system ${ }^{7}$. Faridi et al. (2017) analyzed how inertial effects based on particle size affect the separation of bacteria from blood samples in a microchannel, reporting separation efficiencies up to $76 \%$ and a processing rate of $0.5 \mu \mathrm{L} / \mathrm{min}^{19}$. A study by $\mathrm{Li}$ et al. (2017) analyzed how external acoustic effects applied to a microchannel contribute to bacterial separation from blood in the channel ${ }^{20}$. They used digital transducers to apply acoustic waves to the system and form pressure nodes in the channel that separate $E$. coli from the red blood cells due to the cells' physical properties. They reported a resulting blood purity of $96 \%{ }^{20}$. A study by Wang et al. (2012) used antibody coated channels and an incubation time of 30 minutes to identify fluorescent $E$. coli, with capture efficiencies of $71.8 \%{ }^{21}$. The majority of the bacterial separation work using microchannels does not use any magnetic separation techniques. Instead, these studies opt for separation techniques that do not require the target entity to be bound to mMBs. While this strategy removes a step in the sample preparation process, it limits the specificity and adaptability of the separation process. The use of mMBs allows for the targeted capture of a specific bacterial species and differentiation between multiple types of bacteria in a sample.

Proposed design. Efficient capture of $\mathrm{mMBs}$ and ability to be selective in bacteria targeting are important for the sensitivity and effectiveness of any proposed lab-on-chip device. Our group has developed a $\mu$ TAS device that uses electroosmotic flow (EOF) and fluorescent microscopy to capture mMBs and quantify their concentration in a microfluidic system. The use of EOF is preferred to pressure driven flow due to it being inexpensive and efficient when operating at small volumes while providing improved control over flow rate and direction ${ }^{22,23}$. The developed $\mu$ TAS device takes advantage of EOF by using a flow switching system to increase capture efficiency $\left(\eta_{c}\right)$ by returning uncaptured beads to the area of the channel with higher magnetic field strength. This device has shown capture efficiencies of fluorescent mMBs (mMB* complexes) under switching flow of up to $85 \%$ while analyzing concentrations as low as $10^{6}$ beads $/ \mathrm{mL}^{24}$.

The previous study only analyzed $\mathrm{mMB}^{*}$ s in the system ${ }^{24}$, while this study takes the next step in developing the system for bacterial analysis under realistic scenarios. This work is a novel extension on the previous study due to the introduction of the bacterial pathogen as the fluorescent entity of interest. The previously built device is used to capture and identify magnetic microbead-fluorescent bacteria (mMB-E. coli*) complexes under realistic test scenarios using very small sample volumes. This research is significant because it will allow the assessment of capture efficiency for screening different types of water-borne pathogens while requiring a much smaller sample volume (around 30\% volume) than other devices reported in literature. It was hypothesized that the devices would be able to effectively capture mMB-E. coli* complexes, while allowing for: (1) the creation of a calibration curve of fluorescent intensity as a function of a wide range of $m M B$-E. coli* concentration and (2) comparison of capture efficiency for constant and switching flows. This would prove the usefulness of the system as a point-of-care $\mu$ TAS device for high-throughput screening of pathogens.

\section{Methods}

This section describes the methods used to create the device, the materials used, the design of the experiment, and the analysis performed. In the experiments, $\mathrm{mMB}$-secondary antibody-fluorescent bacteria complexes (mMB-E.

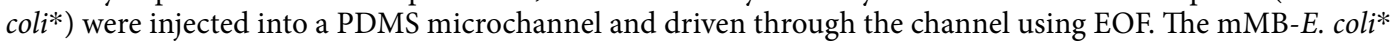
complexes were immobilized in the channel using an external magnet and the fluorescence was characterized using inverted fluorescent microscopy. The captured images were analyzed using MATLAB to determine the captured and uncaptured fluorescent intensities. The capture efficiency was calculated for both switching and 

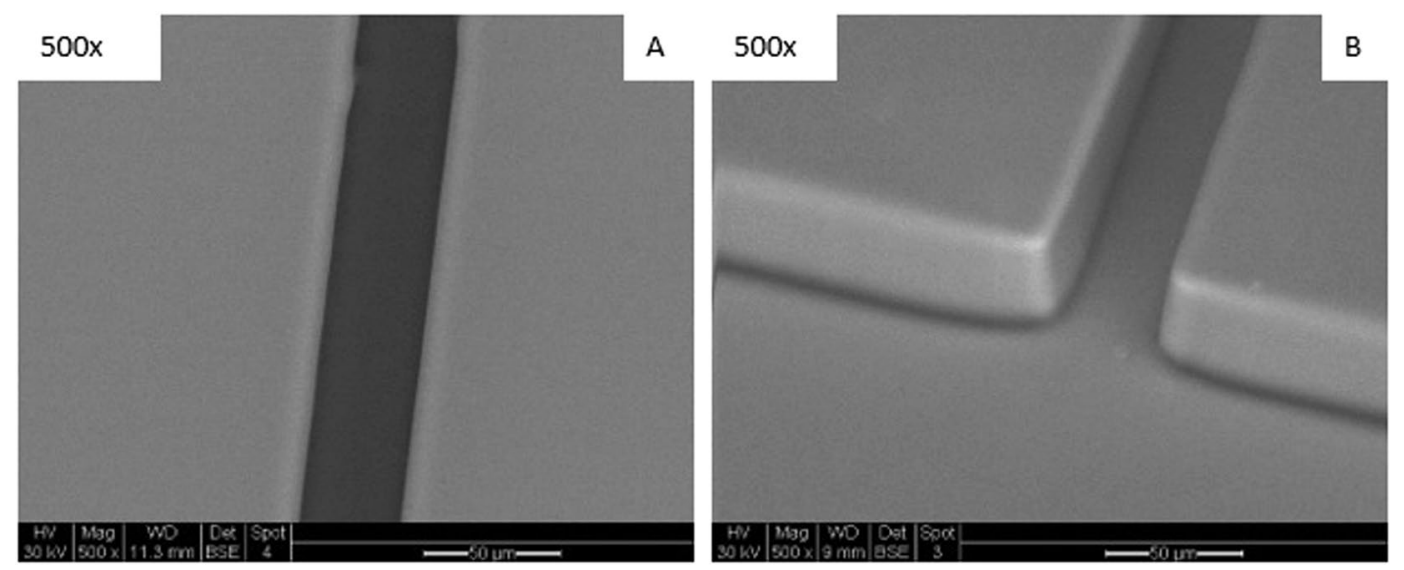

Figure 1. SEM images of $(\mathbf{A})$ a top down view of the microchannel and $(\mathbf{B})$ a tilted view of the entrance to the microchannel from a well.
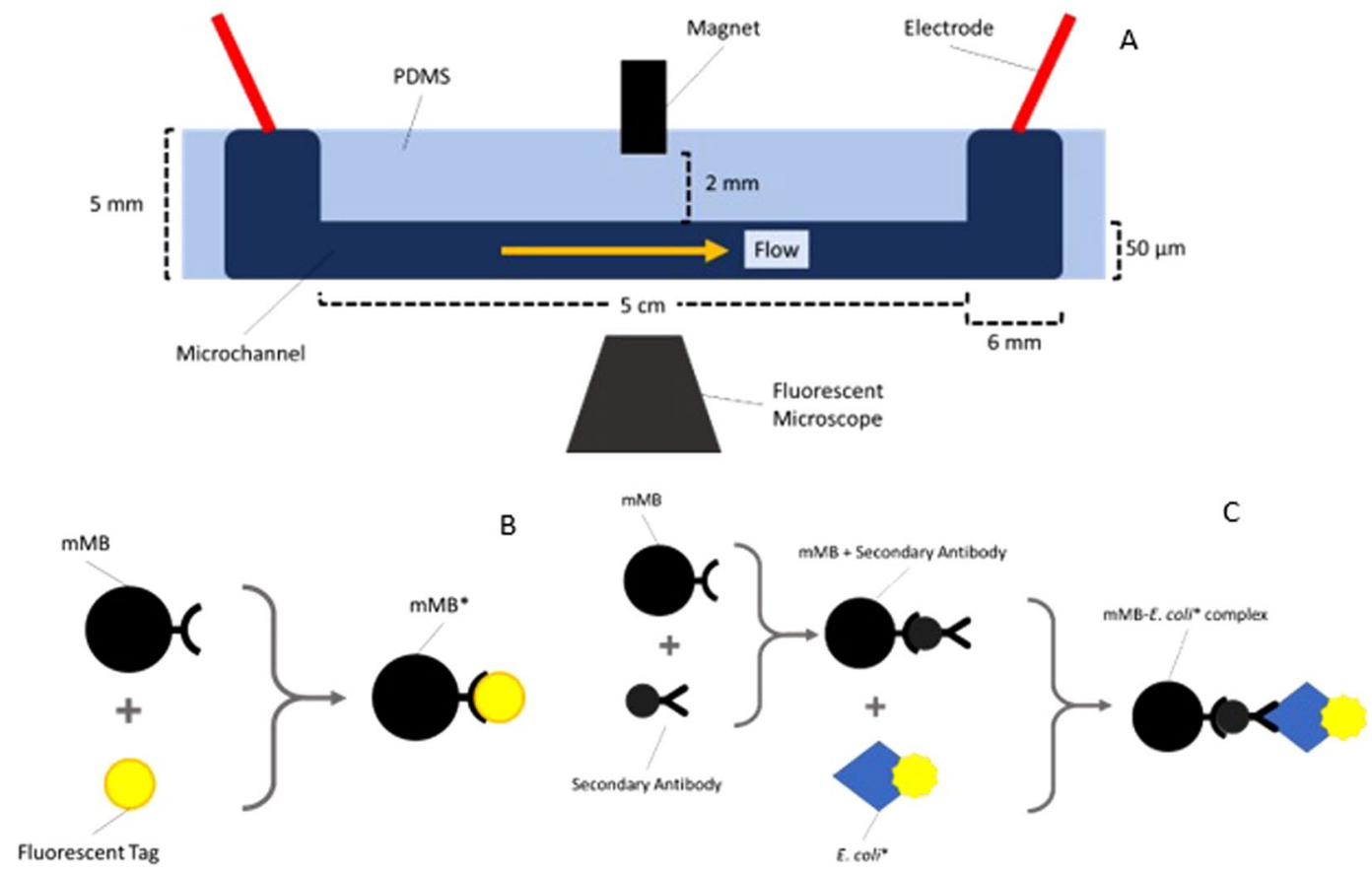

Figure 2. (A) Schematic of the microchannel testing system, (B) schematic of the $\mathrm{mMB}^{*}$ system ${ }^{24}$, and $(\mathbf{C})$ schematic of the mMB-E. coli* system used for E. coli capture.

constant flow protocols. These protocols were compared based on relative percentage difference, and a calibration curve was created based on total fluorescent intensity.

Material properties. The microfluidic device was created following the protocol outlined in the previous publication ${ }^{24}$. In brief, the device was made of PDMS bound to a glass slide to create a $50 \mathrm{~mm}$ long microchannel with a $50 \mu \mathrm{m} \times 50 \mu \mathrm{m}$ cross section with wells at each end of the channel. SEM images of the PDMS channel and one of the wells are shown in Fig. $1 \mathrm{~A}, \mathrm{~B}$. A $1 / 8^{\prime \prime} \times 1 / 8^{\prime \prime} \times 3 / 8^{\prime \prime}$ volume neodymium $(\mathrm{NdFeB})$ magnet was placed above the microchannel halfway between the two wells. A diagram of the experimental setup is shown in Fig. 2A.

The mMBs used in the experiments were $2.8 \mu \mathrm{m}$ diameter Dynabead M280 Sheep anti-Rabbit IgG mMBs (Life Technologies, NY). These mMBs are bound to inherently fluorescent E. coli (E. coli*) by a secondary Virustat anti-E. coli rabbit polyclonal antibody. A schematic of the fluorescent mMB complex (mMB*) without $E$. coli is shown in Fig. 2B whereas a schematic of the new mMB-E. coli* complex is shown in Fig. 2C. The mMB* complexes were created by binding M280 sheep anti-rabbit Dynabeads with Alexa Fluor 488 rabbit anti-mouse fluorescent tag following the procedure described in the previous publication ${ }^{24}$. The mMB-E. coli* complexes were created by incubating $0.4 \mathrm{~mL}$ of $E$. coli*, with a concentration of about $6 \times 10^{8}$ bacteria $/ \mathrm{mL}$, with $0.6 \mathrm{~mL}$ of Dynabeads, with a concentration of $7 \times 10^{8}$ beads $/ \mathrm{mL}$, and excess secondary antibody by slow tilt mixing for 
24 hours at $8^{\circ} \mathrm{C}$. The mMB-E. coli* solution was then diluted with phosphate buffer (PBS, pH 7.5) to achieve the desired concentration for each experiment.

Experimental method. To ensure adequate flow when the external voltage was applied, the channels were treated with $1 \mathrm{M} \mathrm{NaOH}$ solution for 10 minutes. The channel was then washed with PBS buffer and, prior to each experiment, was primed with a $1 \%$ Tween 20 in PBS buffer solution for 5 minutes to decrease the surface tension and prevent the mMBs from sticking to the channel walls while not captured.

After channel preparation, the mMB-E. coli* solution was injected into the inlet well and the chip was placed on the inverted fluorescent microscope. The magnet was put in place and two platinum electrodes were connected to a high voltage sequencer (LabSmith, CA) and placed into the inlet and outlet wells. The experiments were performed under two previously published flow conditions, a switching flow protocol and a constant flow protocol ${ }^{24}$, which are described in further detail below. Under both protocols, the flow was driven at $650 \mathrm{~V}$ EOF voltage. The microscope captured images using MetaMorph Software (Molecular Devices, PA).

The $650 \mathrm{~V}$ flow rate is used to determine the maximum distance before and after the magnet where mMBs are captured. To determine the capture zone, samples were driven through the channel at the lower limit of the voltage. Immediately following the experimental time, the channel was washed with pure PBS buffer with the magnet still in place. This removed any uncaptured $\mathrm{mMBs}$ from the channel so that the maximum distance before and after the magnet that contained $\mathrm{mMBs}$ was determined to be the capture zone. Thus, when performing experiments with mMB-E. coli* complexes, any mMB-E. coli* found in the capture zone are considered captured while those mMB-E. coli* complexes that are found after the capture zone or in the outlet well are considered uncaptured. The mMB-E. coli* complexes that reside in the inlet well or in the channel before the capture zone are not considered as they never entered the zone of magnetic field generated by the stationary magnet and, thus, never entered the experiment.

Characterization. The purpose of this device is to identify mMB-E. coli* concentration in an unknown sample, thus the development of a calibration curve for the device is required. The calibration curve plots total fluorescent intensity as a function of concentration. The total fluorescent intensity of a sample with unknown mMB-E. coli* complex concentration can be measured and compared to the curve to determine the mMB-E. coli* concentration. The total fluorescent intensity is determined by the sum of the fluorescent intensities of all captured mMB-E. coli* complexes in a single experiment. In addition, the capture efficiency $\left(\eta_{c}\right)$ and relative percentage difference between the constant and switching capture efficiencies were calculated. The capture efficiency $\left(\eta_{c}\right)$ was calculated according to Eq. (1) and is based on the ratio of the captured mMB-E. coli* complexes to the total number of mMB-E. coli* complexes that entered the channel based on fluorescent pixel count.

$$
\eta_{\mathrm{c}}=\frac{\text { pixel count captured }{ }_{\mathrm{mMB}-E . \text { coli } *}}{\text { pixel count captured }{ }_{\mathrm{mMB}-E . \text { coli } *}+\text { pixel count uncaptured }{ }_{\mathrm{mMB}-E \text {. coli } *}}
$$

The relative percentage difference between the constant and switching capture efficiency at each concentration was calculated according to Eq. (2),

$$
\text { Relative \% Difference }=\frac{\eta_{c_{-} \text {switching }}-\eta_{c_{-} \text {constant }}}{\eta_{c_{-} \text {constant }}} \times 100 \%
$$

Statistical analysis was then performed using a t-test, where $\mathrm{p}<0.05$ is considered statistically significant.

Constant flow and switching flow protocols. The switching flow protocol experiments are performed under $650 \mathrm{~V}$ EOF flow for 8 minutes forward followed by two periods of alternating 3 minutes backward and then 3 minutes forward for a 20 minute total testing time. The voltages as a function of time at the inlet and outlet of the channel for the switching flow profile are shown in Fig. 3A,B, respectively. The flow rate during one of these periods can be calculated according to the Helmholtz-Smoluchowski equation, as shown in Eq. (3),

$$
\mathrm{U}_{\mathrm{ep}}=-\frac{\mathrm{E}_{\mathrm{z}} \varepsilon_{\mathrm{r}} \varepsilon_{\mathrm{o}} \zeta_{\mathrm{p}}}{\mu}
$$

where $U_{\text {ep }}$ is the velocity $(\mathrm{cm} / \mathrm{s}), \mathrm{E}_{\mathrm{z}}$ is the applied electric field $(\mathrm{V} / \mathrm{cm}), \varepsilon_{\mathrm{r}}$ is the dielectric constant of the medium, $\varepsilon_{\mathrm{o}}$ is the vacuum permittivity $(\mathrm{F} / \mathrm{m}), \zeta_{\mathrm{p}}$ is the zeta potential $(\mathrm{V})$, and $\mu$ is the dynamic viscosity $(\mathrm{Pa} \cdot \mathrm{s})$. For the $650 \mathrm{~V}$ case, $\mathrm{E}_{\mathrm{z}}$ is $130 \mathrm{~V} / \mathrm{cm}, \varepsilon_{\mathrm{r}}$ is $80.4, \varepsilon_{\mathrm{o}}$ is $8.55 \times 10^{-12} \mathrm{~F} / \mathrm{m}, \zeta_{\mathrm{p}}$ is $-95.6 \mathrm{mV}$, and $\mu$ is $8.6 \times 10^{-4} \mathrm{~Pa} \cdot \mathrm{s}$ [Das et al. 2016]. The fluid velocity at this voltage was determined to be $0.099 \mathrm{~cm} / \mathrm{s}$, which equates to a volumetric flow rate of $0.15 \mu \mathrm{L} / \mathrm{min}$. For a 20 minute testing period, this system will process a $3 \mu \mathrm{L}$ sample. With a $5 \mathrm{~cm}$ long channel, a 3 minute period would allow for about 3.5 full channel length $(5 \mathrm{~cm})$ clearances. Thus, this EOF voltage provides ample time for multiple passes of mMB-E. coli* complexes through the channel during each testing period.

During the 20 minute switching flow test, the first 8 minutes of forward flow will introduce the vast majority of mMB-E. coli* complexes to the system, since the subsequent reversals of flow will alternate these mMB-E. coli* complexes flowing backward and forward. Therefore, in order to better compare the total fluorescent intensities between the constant and switching flow protocols for the calibration curve, an 8 minute testing time was used for the constant flow protocol. The 8 minute constant flow time corresponds to the 8 minutes of forward flow for the switching protocol since the oscillating portion of the switching flow protocol is primarily used to increase the residence time of the mMB-E. coli* complexes in the area of higher magnetic field strength within the channel. 


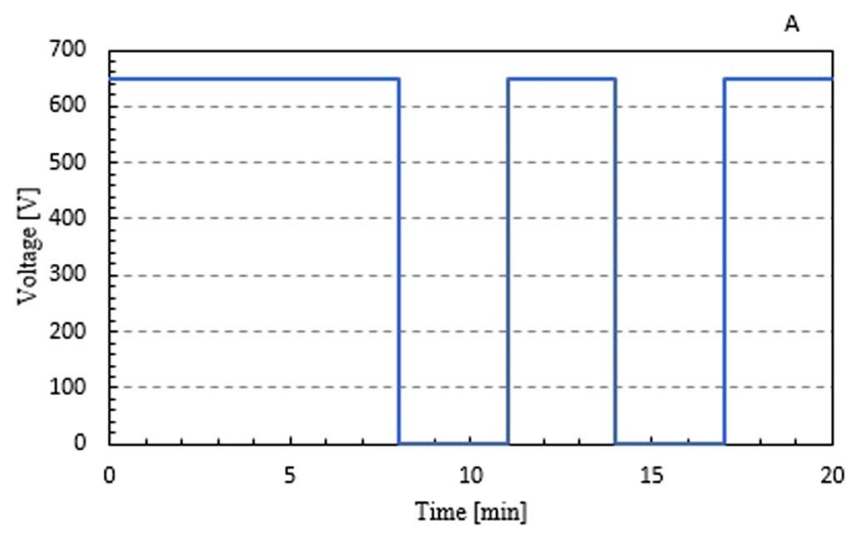

B

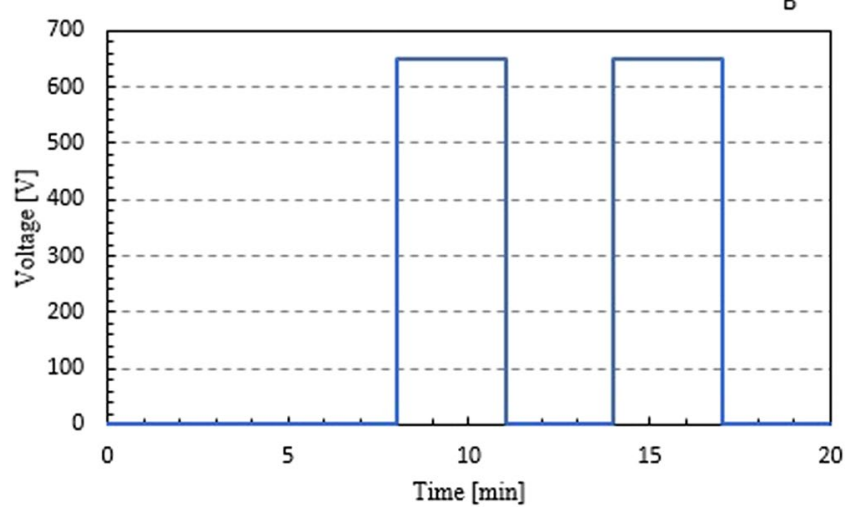

Figure 3. (A) Inlet voltage as a function of time and (B) outlet voltage as a function of time for the switching flow protocol.

This switching allows complexes that may have escaped capture on the first pass enough time to travel the transverse distance along the cross section of the channel and be captured.

\section{Results}

The microfluidic device was tested using mMB-E. coli* complexes concentrations of $2 \times 10^{5}, 2 \times 10^{6}, 4 \times 10^{6}$, $2 \times 10^{7}$, and $4 \times 10^{7}$ bacteria/mL under switching and constant flow protocols at an EOF voltage of $650 \mathrm{~V}$. Fluorescent images were taken to determine a calibration curve and the capture efficiency based on fluorescent intensity of mMB-E. coli* complexes.

The mMB-E. coli* tagging. Solutions with mMB-E. coli* complexes concentrations of $2 \times 10^{5}, 2 \times 10^{6}$, $4 \times 10^{6}, 2 \times 10^{7}$, and $4 \times 10^{7}$ bacteria/mL were tested in a microfluidic device using both the constant and switching flow protocols at an EOF voltage of $650 \mathrm{~V}$. Fluorescent images were taken along the length of the channel for each concentration, with images in the capture zone determined to be captured and images after the capture zone counted as uncaptured. In order to ensure that mMB-E. coli* complexes were forming and that the E. coli* were the only fluorescent entity in the system, images were taken of the same area with and without an additional light source. This light source shines white light on the channel along with the exciting laser required for fluorescence. This allows viewing of the fluorescent entity while also illuminating the mMBs in the system via the white (secondary) light. Figure 4 shows sample taken in the channel with and without this light source. Figure 4A,B show the same area of the channel with and without the white light source whereas Fig. 4C,D show a different area with and without the white light source. These comparative images demonstrate that any unbonded mMBs will not affect the laser (primary light) induced fluorescent images in the condition without secondary white light used in the experiments. This is because the E. coli* portion of the mMB- E. coli* complexes are the only (primary) fluorescent entities.

Calibration curve. The mMB-E. coli* complexes were analyzed in the device to create a calibration curve of fluorescent intensity as a function of mMB-E. coli* concentration. The mMB-E. coli* complexes were delivered through the system at concentrations of $2 \times 10^{5}, 2 \times 10^{6}, 4 \times 10^{6}, 2 \times 10^{7}$, and $4 \times 10^{7}$ bacteria/mL and an EOF voltage of $650 \mathrm{~V}$. For this analysis, only the images of captured mMB-E. coli* complexes were used to determine the fluorescent intensity. The calibration curve, shown in Fig. 5, shows fluorescent pixel count as a function of concentration on a log-log plot. Figure $6 \mathrm{~A}, \mathrm{~B}$ show the calibration curve on a standard axes plot, with a series of linear fits shown in Fig. 6A and a power law fit shown in Fig. 6B. Both the switching and constant flow protocols result in a linearly increasing trendline on the $\log$ - $\log$ plot, with $\mathrm{R}^{2}$ values of 0.99 for both the switching and constant cases. There is a significant difference between the fluorescent intensities at each concentration for both the 

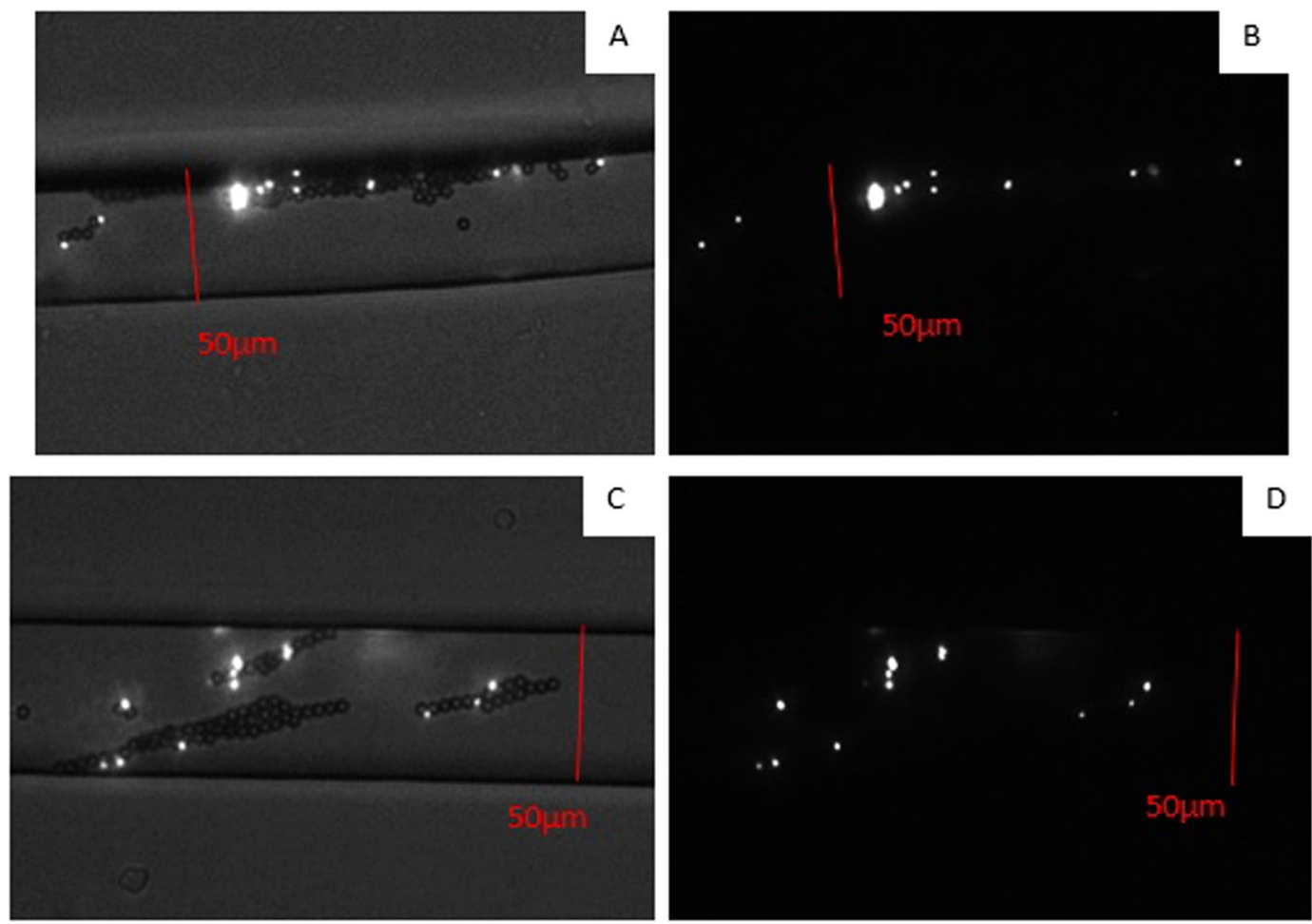

Figure 4. Fluorescent images that captured mMB-E. coli* complexes with $(\mathbf{A})$ and $(\mathbf{B})$ showing a different area and $(\mathbf{C})$ and $(\mathbf{D})$ showing the same area. $(\mathbf{A})$ and $(\mathbf{C})$ are taken using the external light source to show both the E. coli* and the attached mMBs, while (B) and (D) are taken under the dark condition used during experiments showing only the E. coli* fluorescence. The width of the channel is $\sim 50 \mu \mathrm{m}$.

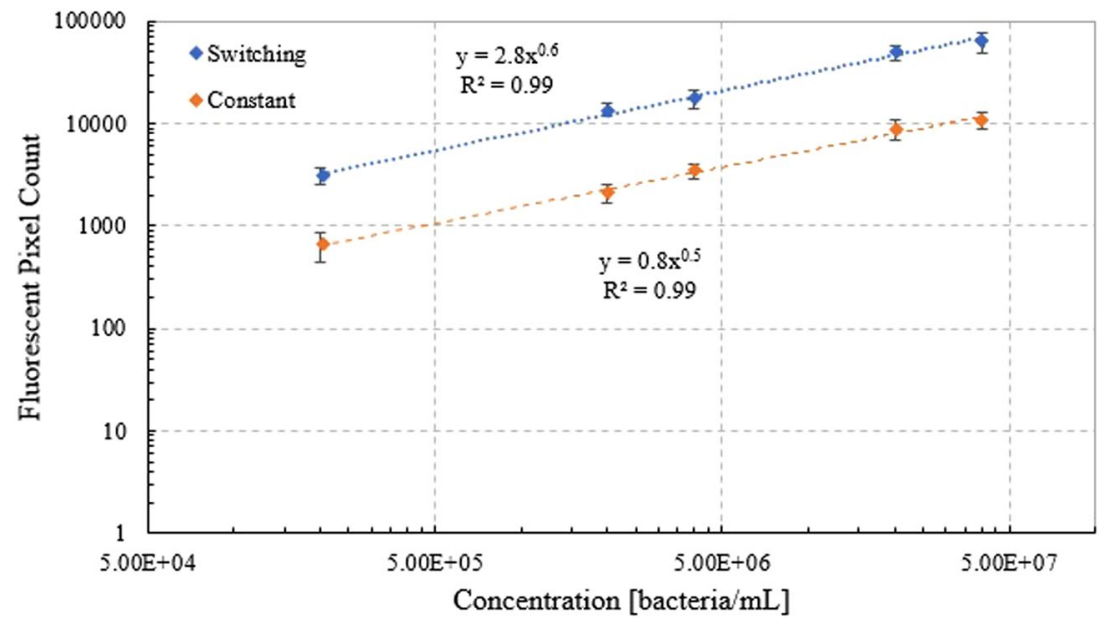

Figure 5. Calibration curve of fluorescent intensity as a function of concentration for capture of mMB-E. coli* complexes in a microchannel under the switching flow protocol at $650 \mathrm{~V}$ on log-log scale plot.

switching and constant flow protocols with relative differences ranging between 3.7 times $\left(e . g\right.$. , $\frac{3070-660}{660} \times 100 \%$ for concentration of $2 \times 10^{5}$ bacteria/ $\mathrm{mL}$ ) to 5.6 times $\left(\right.$ e. $g$. , $\frac{13560-2070}{2070} \times 100 \%$ for concentration of $2 \times 10^{6}$ bacteria $/ \mathrm{mL}$ ) the constant flow values, and $\mathrm{p}<0.05$ for each case. For the constant flow protocol curve, $\mathrm{p}<0.05$ when comparing fluorescent intensity values for neighboring concentrations, except when comparing the fluorescent pixel count between $2 \times 10^{7}$ and $4 \times 10^{7}$ bacteria $/ \mathrm{mL}(\mathrm{p} \approx 0.35)$. This is also the case when comparing neighboring concentrations on the switching flow curve except when comparing the fluorescent pixel count between $2 \times 10^{6}$ and $4 \times 10^{6}$ bacteria $/ \mathrm{mL}(\mathrm{p} \approx 0.15)$, and then also between $2 \times 10^{7}$ and $4 \times 10^{7}$ bacteria $/ \mathrm{mL}(\mathrm{p} \approx 0.22)$.

Comparison between constant and switching flow of mMB-E. coli*. The capture efficiency $\left(\eta_{c}\right)$ and relative percentage difference for $\mathrm{mMB}-E$. coli* between the constant and switching cases at each concentration 


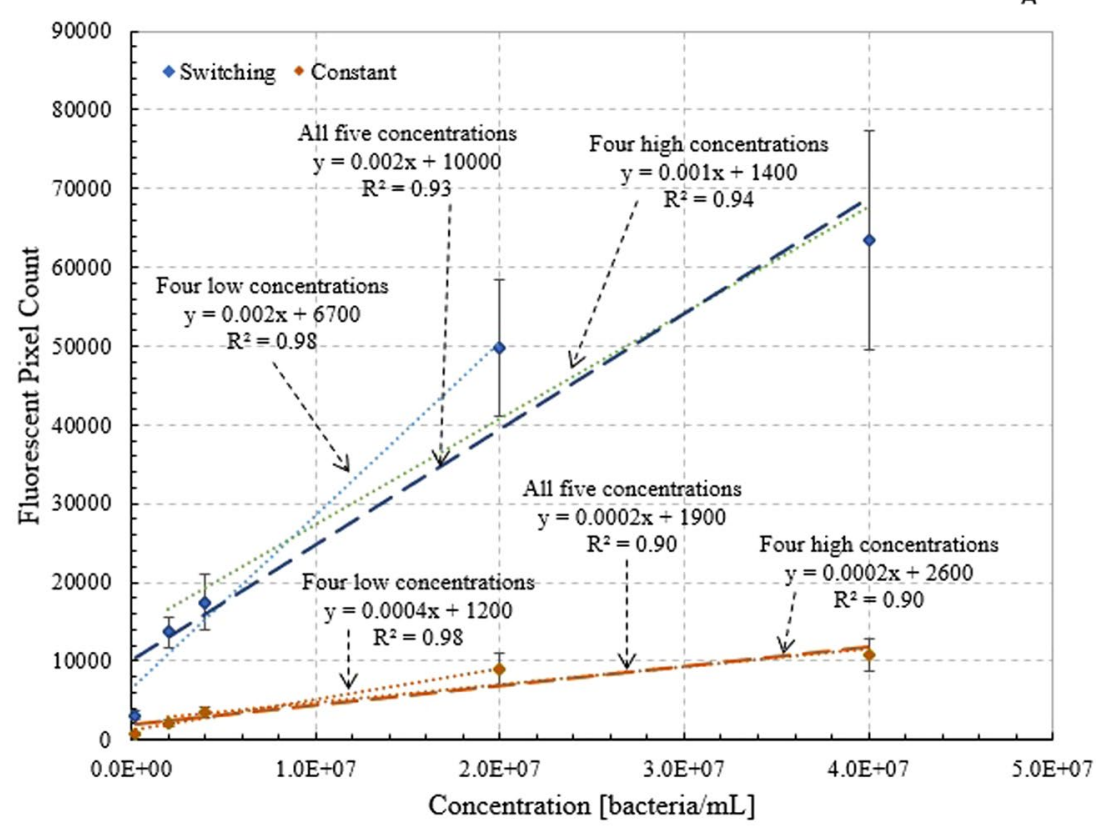

B

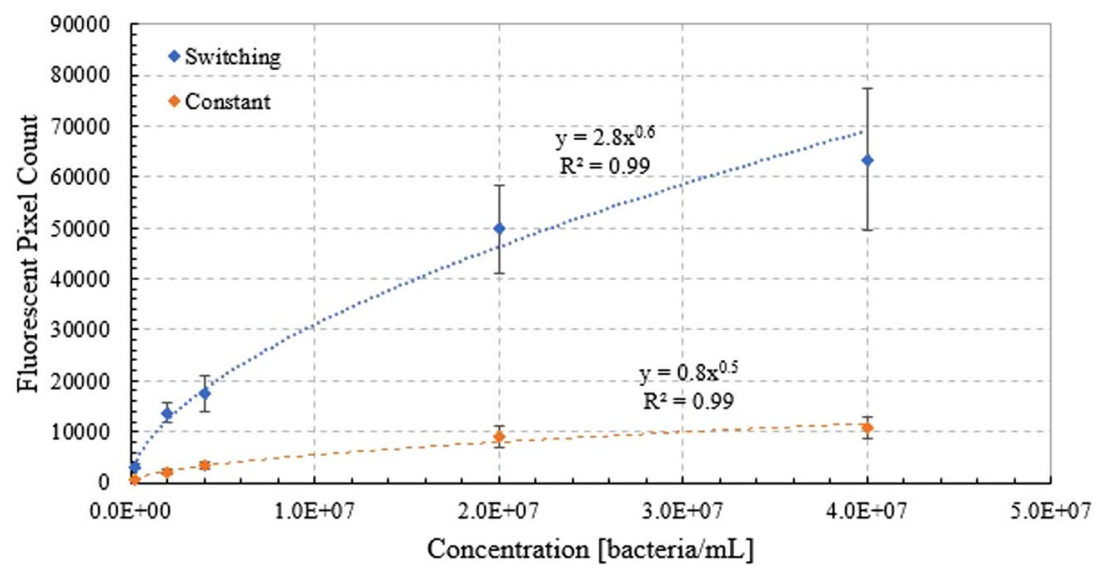

Figure 6. Calibration curve of fluorescent intensity of captured mMB-E. coli* complexes as a function of concentration in the microfluidic device under the constant flow protocol and EOF voltage of $650 \mathrm{~V}$ with standard scale axes using a (A) series of linear trendlines and (B) power law fit.

were also determined. The $\eta_{\mathrm{c}}$ were compared for mMB-E. coli* complexes at concentrations of $2 \times 10^{5}, 2 \times 10^{6}$, $4 \times 10^{6}, 2 \times 10^{7}$, and $4 \times 10^{7}$ bacteria/mL under both constant and switching flow protocols, as shown in Fig. 7 , with $\mathrm{p}<0.05$ at all concentrations. The values for captured, uncaptured, and total pixel count as well as the capture efficiency for the constant and switching cases are reported in Tables 1 and 2, respectively. The $\eta_{\mathrm{c}}$ for $\mathrm{mMB}-E$. coli* complexes that are reported are calculated by averaging the capture efficiency values under the individual flow conditions. The $\eta_{\mathrm{c}}$ under the constant flow protocol were overall lower (compared to switching flow case; see below) at $36 \%, 39 \%, 34 \%, 34 \%$, and $38 \%$ for concentrations of $2 \times 10^{5}, 2 \times 10^{6}, 4 \times 10^{6}, 2 \times 10^{7}$, and $4 \times 10^{7}$ bacte$\mathrm{ria} / \mathrm{mL}$. In contrast, the switching flow protocol $\eta_{\mathrm{c}}$ were overall significantly higher (compared to constant flow case; see above) at $72 \%, 83 \%, 73 \%, 78 \%$, and $81 \%$ for the same concentrations. The relative difference in $\eta_{\mathrm{c}}$ between switching and constant flows were about 2 times $\left(\right.$ e.g., $\frac{72-36}{36} \times 100 \%$ at concentrations of $2 \times 10^{5}$ bacteria/mL) for all mMB-E. coli* concentrations.

Comparison between $\mathrm{mMB} *$ and $\mathrm{mMB}-\mathrm{E}$. coli for all flow protocols. The $\eta_{\mathrm{c}}$ of mMB-E. coli* complexes for constant and switching flow protocols at $2 \times 10^{6}$ and $4 \times 10^{6}$ bacteria/mL were compared to the $\eta_{\mathrm{c}}$ of only mMB* complexes (without E. coli) ${ }^{24}$, as shown in Fig. 8. For the comparison between $\eta_{\mathrm{c}}$ of $\mathrm{mMB}^{*}$ and mMB-E. Coli* complexes at concentrations of $2 \times 10^{6}$ bacteria (or bead for mMB* complex $\eta_{\mathrm{c}}$ ) $/ \mathrm{mL}$ under constant flow, a $\eta_{\mathrm{c}}$ of $39 \%$ was reported for the mMB-E. coli* complexes while a $\eta_{\mathrm{c}}$ of $42 \%$ was reported for the $\mathrm{mMB}^{*}$ complexes. Likewise, for the $4 \times 10^{6}$ concentration under constant flow, a $\eta_{\mathrm{c}}$ of $34 \%$ was reported for the mMB-E. coli* complexes while a $\eta_{\mathrm{c}}$ of $36 \%$ was reported for the $\mathrm{mMB}^{*}$ complexes. For the $2 \times 10^{6}$ concentration under switching flow, a $\eta_{\mathrm{c}}$ of $83 \%$ was reported for the mMB-E. coli* complexes while a $\eta_{\mathrm{c}}$ of $85 \%$ was reported 


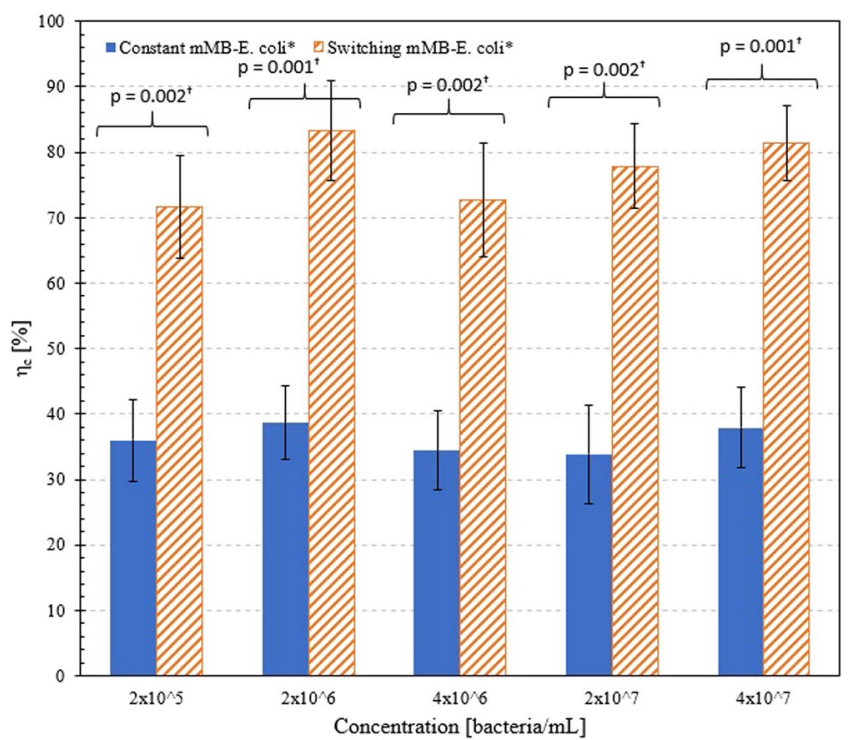

Figure 7. Capture efficiency of mMB-E. coli* complexes under switching flow based on fluorescent pixel counts at $650 \mathrm{~V}$. ( ${ }^{\dagger}$ indicates statistical significance, $\left.\mathrm{p}<0.05\right)$.

\begin{tabular}{|l|c|l|l|l|}
\hline Conditions & $\begin{array}{l}\text { Captured Pixel } \\
\text { Count } \pm \text { SD }\end{array}$ & $\begin{array}{l}\text { Uncaptured Pixel } \\
\text { Count } \pm \text { SD }\end{array}$ & $\begin{array}{l}\text { Total Pixel } \\
\text { Count } \pm \text { SD }\end{array}$ & $\begin{array}{l}\text { Capture Efficiency } \\
\left(\eta_{c}\right) \pm \text { SD }\end{array}$ \\
\hline $2 \times 10^{5}$ & $660 \pm 210$ & $1150 \pm 150$ & $1810 \pm 180$ & $36 \pm 9 \%$ \\
\hline $2 \times 10^{6}$ & $2070 \pm 430$ & $3500 \pm 1400$ & $5570 \pm 1570$ & $39 \pm 11 \%$ \\
\hline $4 \times 10^{6}$ & $3440 \pm 620$ & $6810 \pm 2050$ & $10250 \pm 1680$ & $34 \pm 10 \%$ \\
\hline $2 \times 10^{7}$ & $8920 \pm 2060$ & $18010 \pm 6050$ & $26940 \pm 6050$ & $34 \pm 9 \%$ \\
\hline $4 \times 10^{7}$ & $10730 \pm 2020$ & $18320 \pm 5810$ & $29050 \pm 4980$ & $38 \pm 11 \%$ \\
\hline
\end{tabular}

Table 1. Average capture efficiencies and pixel counts for the constant flow cases. The reported capture efficiency is calculated from the average of the individual capture efficiencies.

\begin{tabular}{|l|l|l|l|l|}
\hline Conditions & $\begin{array}{l}\text { Captured Pixel } \\
\text { Count } \pm \text { SD }\end{array}$ & $\begin{array}{l}\text { Uncaptured Pixel } \\
\text { Count } \pm \text { SD }\end{array}$ & $\begin{array}{l}\text { Total Pixel } \\
\text { Count } \pm \text { SD }\end{array}$ & $\begin{array}{l}\text { Capture Efficiency } \\
(\eta \mathbf{c}) \pm \text { SD }\end{array}$ \\
\hline $2 \times 10^{5}$ & $3070 \pm 580$ & $1290 \pm 700$ & $4360 \pm 1270$ & $72 \pm 8 \%$ \\
\hline $2 \times 10^{6}$ & $13560 \pm 1890$ & $2860 \pm 1760$ & $16510 \pm 3030$ & $83 \pm 8 \%$ \\
\hline $4 \times 10^{6}$ & $17500 \pm 3610$ & $6440 \pm 1740$ & $23940 \pm 2350$ & $73 \pm 9 \%$ \\
\hline $2 \times 10^{7}$ & $49850 \pm 8670$ & $13860 \pm 3290$ & $63710 \pm 5960$ & $78 \pm 6 \%$ \\
\hline $4 \times 10^{7}$ & $63430 \pm 13980$ & $13970 \pm 2650$ & $77410 \pm 11950$ & $81 \pm 6 \%$ \\
\hline
\end{tabular}

Table 2. Average capture efficiencies and pixel counts for the switching flow cases. The reported capture efficiency is calculated from the average of the individual capture efficiencies.

for the $\mathrm{mMB}^{*}$ complexes. Finally, for the $4 \times 10^{6}$ concentration under switching flow, a $\eta_{\mathrm{c}}$ of $73 \%$ was reported for the mMB-E. coli* complexes while a $\eta_{\mathrm{c}}$ of $80 \%$ was reported for the $\mathrm{mMB}^{*}$ complexes. For both constant and switching cases, $\mathrm{p}>0.05$ when comparing the mMB-E. coli* results to the $\mathrm{mMB}^{*}$ results. This indicates that there is no significant difference between $\eta_{\mathrm{c}}$ when working with $\mathrm{mMB} *$ complexes only and $\mathrm{mMB}-E$. coli* complexes.

\section{Discussion}

This study analyzes the ability of a microfluidic device to detect and quantify the concentration of mMB-E. coli* complexes in water samples. The microfluidic device was tested using mMB-E. coli* complex concentrations of $2 \times 10^{5}, 2 \times 10^{6}, 4 \times 10^{6}, 2 \times 10^{7}$, and $4 \times 10^{7}$ bacteria/mL under switching and constant flow protocols at an EOF voltage of $650 \mathrm{~V}$. Fluorescent images were analyzed in order to determine a calibration curve of fluorescent intensity based on mMB-E. coli* complex concentration. Consequently, the capture efficiencies of these mMB-E. coli* complexes in the system were determined.

Bacteria tagging. The ability to tag non-fluorescent mMBs to E. coli* is an important step for testing the operation of the device under real conditions. While the previous experiment ${ }^{24}$ used $\mathrm{mMB}^{*}$ complexes to test the microfluidic device, this study evaluated the operation of the device under realistic scenarios by attempting 


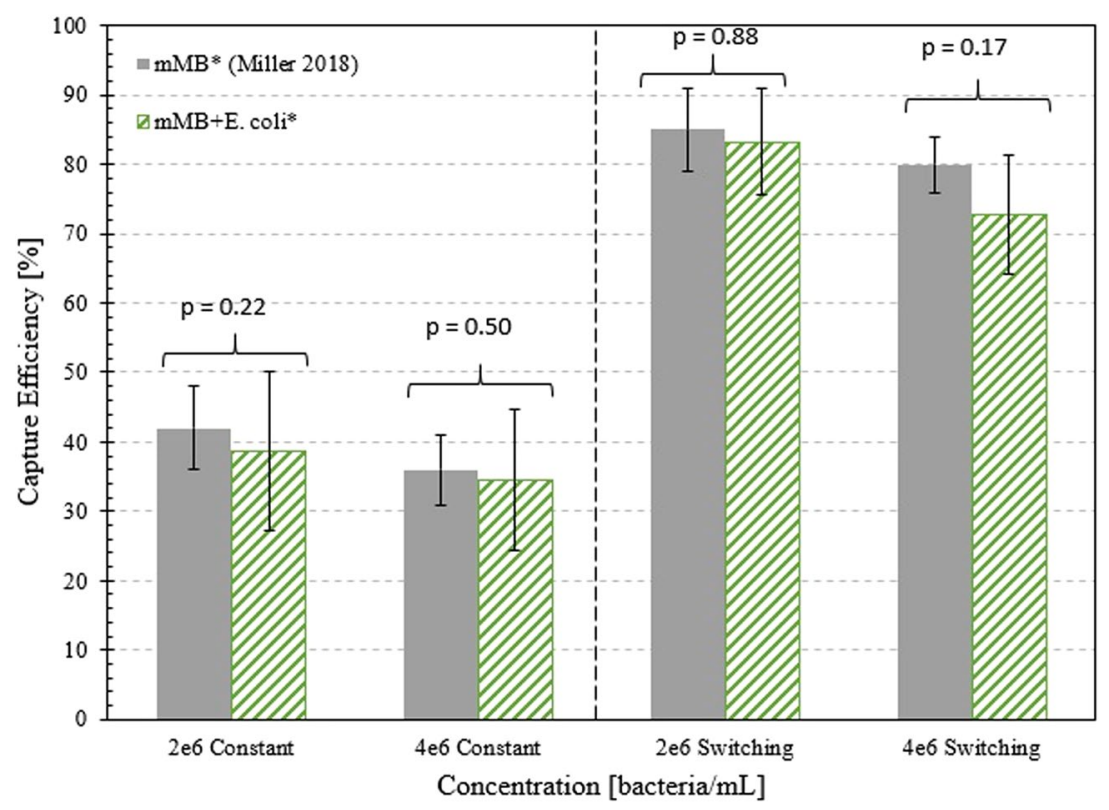

Figure 8. Comparison of capture efficiency of mMB* complexes $^{24}$ and mMB-E. coli* complexes under constant and switching flow $2 \times 10^{6}$.

to identify E. coli* in a water sample through the coupling of E. coli* with mMBs. To minimize the artifact in the device, the $E$. coli* must be the only fluorescent entity in the system. This study used a secondary binding antibody that would only allow E. coli* to bind to the mMBs, thus preventing the creation of fluorescent mMB $\left(\mathrm{mMB}^{*}\right)$ complexes. Consequently, excess mMBs are used to ensure increased sites for coupling of mMBs with the E. coli*; thereby avoiding formation of $\mathrm{mMB}^{*}$ complexes.

Fluorescent calibration curve. Samples with mMB-E. coli* complex concentrations of $2 \times 10^{5}, 2 \times 10^{6}$, $4 \times 10^{6}, 2 \times 10^{7}$, and $4 \times 10^{7}$ bacteria/mL were analyzed in the microfluidic device under an EOF voltage of $650 \mathrm{~V}$ using both the switching and constant flow protocols. A significant increase in fluorescent intensity is seen as the mMB-E. coli* complex concentration increases from $2 \times 10^{5}$ to $4 \times 10^{7}$ bacteria/mL using both the switching and constant flow protocols. More importantly, the fluorescent intensity under the switching flow protocol is significantly higher than under the constant flow protocol. This increase in fluorescence is due to the increase in residence time within the channel that experiences high magnetic field strength. The reversal of the flow in the switching protocol allows for $\mathrm{mMBs}$ that remained uncaptured after the first pass to return through the channel, creating the increased residence time in the channel. This allows for an increase in the number of mMB-E. coli* complexes captured and, therefore, the total fluorescence in the channel.

It is interesting to note that while the fluorescent pixel count in the calibration curve does follow a linear trend, it also exhibits a power law relationship with the exponent less than one. This levelling out of the curve could occur due to the limited confines of the channel creating overlap of fluorescent entities at higher concentrations. This would cause a dampening of the total fluorescent pixel counts at higher concentrations, creating the plateau effect seen in Fig. 6B. Similarly, the presence of additional mMBs in the system could be causing a dampening effect. This effect would become more prevalent at higher concentrations as the total area of the top channel wall (where the beads are captured) is nearing saturation. This would cause any fluorescence beneath them to be suppressed, decreasing to total fluorescent pixel count. Meanwhile, at lower concentrations, the total number of $\mathrm{mMB}-E$. coli* complexes entering the system is lower, so there is a decreased likelihood that any particular fluorescent source would be blocked.

Since this curve relies on the magnetic capture of mMB-E. coli* complexes against the top wall of the channel, variations of known gas adsorption models can be used as possible fits. The Freundlich adsorption model is an empirically derived model presented in Eq. 4

$$
q_{e}=k_{f} C_{e}^{\frac{1}{n}}
$$

where $\mathrm{q}_{\mathrm{e}}$ is the amount of solute adsorbed (gram adsorbate/gram adsorbent), $\mathrm{C}_{\mathrm{e}}$ is the equilibrium adsorbate concentration (gram adsorbate $/ \mathrm{mL}$ ), and $\mathrm{k}_{\mathrm{f}}$ and $1 / \mathrm{n}$ are fitting parameters. This model can be adopted to the current system by equating $\mathrm{q}_{\mathrm{e}}$ to the fluorescent pixel count and $\mathrm{C}_{\mathrm{e}}$ to the bacteria concentration. In this case $1 / \mathrm{n}$ represents the strength of the attraction to the magnet and is similar when comparing the constant and switching flow cases. Meanwhile, $\mathrm{k}_{\mathrm{f}}$ represents the extent of the attraction, with the switching flow case showing a much greater value due to the returning of uncaptured beads, resulting in an increased pixel count ${ }^{25-27}$. The use of the Freundlich model accounts for the asymptotic trend shown in the calibration curve due to the rough surface, non-ideal interactions, and potential overlapping of mMBs and mMB-E. coli* complexes in the system. The 
adoption of the Freundlich model also validates the conclusions derived by the various linear trendlines shown in Fig. 6A. The trendlines show more accurate fits, as indicated by the $\mathrm{R}^{2}$ values closer to 1 , at the lower concentration ranges compared to the higher concentration ranges. This indicates that the calibration curve is more accurate at the lower end of the concentration ranged examined while the sensitivity slightly decreases at higher concentrations.

Capture efficiency under constant and switching flows. The $\eta_{\mathrm{c}}$ of mMB-E. coli* complexes at concentrations of $2 \times 10^{5}, 2 \times 10^{6}, 4 \times 10^{6}, 2 \times 10^{7}$, and $4 \times 10^{7}$ bacteria/mL was also determined based on fluorescent pixel analysis. The $\eta_{\mathrm{c}}$ of mMB-E. coli* complexes under switching flow were approximately 2 times higher than the capture efficiency mMB-E. coli* complexes under constant flow for all concentrations. In addition, the $\eta_{\mathrm{c}}$ for $\mathrm{mMB}^{*}$ complexes only ${ }^{24}$ and mMB-E. coli* complexes were similar under the same flow protocols and concentrations.

Limitations of experiment. One of the limitations of the study is that the exact concentration (or number) of mMB-E. coli* complexes captured could not be counted, instead a relative quantification of fluorescence was reported. The pixel counts based on fluorescent images of the mMB-E. coli* complexes were used to compare captured and uncaptured E. coli*, providing the $\eta_{c}$ for each condition. Additionally, this study is conducted for a single voltage difference of $650 \mathrm{~V}$. The $\eta_{\mathrm{c}}$ needs to be tested for different voltage differences, resulting in varied EOF values. It is likely that increasing the voltage will decrease the capture efficiency due to the increased flow rate. Also, the $\eta_{c}$ needs to be optimized for different channel dimensions (length and width) and shapes. A smaller channel will likely increase capture efficiency due to a smaller maximum distance mMBs can start from the magnet. However, a smaller channel will also decrease the flow rate and number of mMB-E. coli* complexes analyzed as well as increase the potential for clogging in the channel. In addition the $\eta_{c}$ was only analyzed for a stationary magnet instead of electromagnets, which are popular and may be tested for variable magnetic fields within the proposed microfluidic device. Finally, the system was only analyzed using E. coli* as the target cell of interest. It is expected that different calibration curves would be required for different bacterial entities.

Future direction. While this study used an idealistic sample with only E. coli* for calibration purposes, the presence of other biological entities in a realistic sample needs to be further analyzed. Further optimization of the device over a wider range of bacteria concentration also needs to be addressed. The device has been shown to work well in the range considered in this study; however the plateau trend at higher concentrations (seen in Fig. 6) indicates a need to analyze higher concentrations. In addition, analyzing lower bacteria concentrations would further help calibrate the device for some specific bacteria strains such as E. coli O157:H7. In addition, the use of a two-step binding process within the microchannel to create a mMB-bacteria-fluorescent tag complex is necessary for the eventual application of the device. The use of two separate bacteria targeting antibodies, one for the $\mathrm{mMB}$ and one for the fluorescent tag, would prevent competition over binding sites on the bacteria, allowing for improved characterization and quantification of bacteria in a sample. Finally, the analysis of additional voltages between 100-900 V, where Joule heating is at a minimum, needs to be analyzed in order to optimize capture efficiency and accuracy of the device.

\section{Data Availability}

The datasets generated during and/or analyzed during the current study are available from the corresponding author on reasonable request.

\section{References}

1. Manz, A., Graber, N. \& Widmer, H. á Miniaturized total chemical analysis systems: a novel concept for chemical sensing. Sensors and actuators B: Chemical 1, 244-248 (1990).

2. Deisingh, A. K. \& Thompson, M. Biosensors for the detection of bacteria. Canadian journal of microbiology 50, 69-77 (2004).

3. Greig, J. D., Todd, E. C. D., Bartleson, C. \& Michaels, B. Infective doses and pathogen Carriage, 2010 Food Safety Education Conference, 2010

4. McCloskey, K. E., Chalmers, J. J. \& Zborowski, M. Magnetic cell separation: characterization of magnetophoretic mobility. Analytical chemistry 75, 6868-6874 (2003).

5. Choi, J.-W. Fabrication of micromachined magnetic particle separators for bioseparation in microfluidic systems Microfluidic Techniques: Reviews and Protocols, pp. 65-81 (2006).

6. Beyor, N., Seo, T. S., Liu, P. \& Mathies, R. A. Immunomagnetic bead-based cell concentration microdevice for dilute pathogen detection. Biomedical microdevices 10, 909-917 (2008).

7. Kwon, Y. et al. Magnetic bead based immunoassay for autonomous detection of toxins. Analytical chemistry 80, 8416-8423 (2008).

8. Choi, J.-W., Liakopoulos, T. M. \& Ahn, C. H. An on-chip magnetic bead separator using spiral electromagnets with semiencapsulated permalloy. Biosensors and Bioelectronics 16, 409-416 (2001).

9. Gijs, M. A. Magnetic bead handling on-chip: new opportunities for analytical applications. Microfluidics and nanofluidics 1, 22-40 (2004).

10. Guo, S., Deng, Y., Zhao, L., Chan, H. \& Zhao, X. Effect of patterned micro-magnets on superparamagnetic beads in microchannels. Journal of Physics D: Applied Physics 41, 105008 (2008).

11. Thompson, J. A., Du, X., Grogan, J. M., Schrlau, M. G. \& Bau, H. H. Polymeric microbead arrays for microfluidic applications. Journal of Micromechanics and Microengineering 20, 115017 (2010).

12. Di Carlo, D., Irimia, D., Tompkins, R. G. \& Toner, M. Continuous inertial focusing, ordering, and separation of particles in microchannels. Proceedings of the National Academy of Sciences 104, 18892-18897 (2007).

13. Li, P., Stratton, Z. S., Dao, M., Ritz, J. \& Huang, T. J. Probing circulating tumor cells in microfluidics. Lab on a chip 13, 602-609 (2013).

14. Zhang, Z., Xu, J., Hong, B. \& Chen, X. The effects of 3D channel geometry on CTC passing pressure-towards deformability-based cancer cell separation. Lab on a chip 14, 2576-2584 (2014). 
15. Wu, X., Wu, H. \& Hu, Y. Enhancement of separation efficiency on continuous magnetophoresis by utilizing L/T-shaped microchannels. Microfluid Nanofluid 11, 11-24 (2011).

16. Munir, A., Wang, J. \& Zhou, H. Dynamics of capturing process of multiple magnetic nanoparticles in a flow through microfluidic bioseparation system. IET nanobiotechnology 3, 55-64 (2009).

17. Hoshino, K. et al. Microchip-based immunomagnetic detection of circulating tumor cells. Lab on a chip 11, 3449-3457 (2011).

18. Ramadan, Q., Poenar, D. P. \& Yu, C. Customized trapping of magnetic particles. Microfluidics and nanofluidics 6, 53-62 (2009).

19. Faridi, M. A. et al. Elasto-inertial microfluidics for bacteria separation from whole blood for sepsis diagnostics, Journal of Nanobiotechnology, vol 15 (2017).

20. Li, S. et al. Acoustofluidic bacteria separation, Journal of Micromechanics and Microengineering, vol. 27 (2017).

21. Wang, S. Q. et al. Portable microfluidic chip for detection of Escherichia coli in produce and blood. International journal of nanomedicine 7, 2591 (2012).

22. Comandur, K. A., Bhagat, A. A. S., Dasgupta, S., Papautsky, I. \& Banerjee, R. K. Transport and reaction of nanoliter samples in a microfluidic reactor using electro-osmotic flow. Journal of Micromechanics and Microengineering 20, 035017 (2010).

23. Al-Rjoub, M. F., Roy, A. K., Ganguli, S. \& Banerjee, R. K. Enhanced electro-osmotic flow pump for micro-scale heat exchangers, ASME 2012 Third International Conference on Micro/Nanoscale Heat and Mass Transfer pp. 829-833 (2012).

24. Miller, S. A., Heineman, W. R., Weiss, A. A. \& Banerjee, R. K. Analysis of magnetic microbead capture with and without bacteria in a microfluidic device under different flow scenarios. Journal of Medical Devices 12(4), 041005 (2018).

25. Xu, Z. et al. Development of a novel mixed titanium, silver oxide polyacrylonitrile nanofiber as a superior adsorbent and its application for MB removal in wastewater treatment. Journal of the Brazilian Chemical Society 29, 560-571 (2017).

26. Wei, H. et al. Highly flexible herapin-modified chitosan/graphene oxide hybrid hydrogel as a super bilirubin adsorbent with excellent hemocompatibility. Journal of Materials Chemistry B 3, 1646-1654 (2015).

27. Dada, A. O., Olalekan, A. P., Olatunya, A. M. \& Dada, O. Langmuir, Freundlich, Temkin, and Dubinin-Radushkevich isotherms studies of equilibrium sorption of $\mathrm{Zn}^{2+}$ unto phosphoric acid modified rice husk. IOSR Journal of Applied Chemistry 3, 35-45 (2012).

\section{Acknowledgements}

This research was supported by the National Institute for Occupational Safety and Health through the University of Cincinnati Education and Research Center Grant \#T42OH008432.

\section{Author Contributions}

This research was performed by Samuel Miller with Dr. Rupak Banerjee serving as the mentor and supervisor. Dr. Alison Weiss provided the bacteria used during testing as well as assistance in performing the bacteria tagging. Dr. William Heineman provided the equipment and lab space used for the fluorescent microscopy.

\section{Additional Information}

Competing Interests: The device has been submitted for a patent by Dr. Rupak Banerjee. The inventors are: Banerjee R.K., Das D., Al-Rjoub M. and Yadav J. United States Provisional Patent Application EFS ID: 21291639, Application no: 62106883, Publication Date: 01/23/2015. The other authors have no competing interests.

Publisher's note Springer Nature remains neutral with regard to jurisdictional claims in published maps and institutional affiliations.

(c) (i) Open Access This article is licensed under a Creative Commons Attribution 4.0 International

License, which permits use, sharing, adaptation, distribution and reproduction in any medium or format, as long as you give appropriate credit to the original author(s) and the source, provide a link to the Creative Commons license, and indicate if changes were made. The images or other third party material in this article are included in the article's Creative Commons license, unless indicated otherwise in a credit line to the material. If material is not included in the article's Creative Commons license and your intended use is not permitted by statutory regulation or exceeds the permitted use, you will need to obtain permission directly from the copyright holder. To view a copy of this license, visit http://creativecommons.org/licenses/by/4.0/.

(C) The Author(s) 2019 